\title{
Documentation Disillusionment
}

\author{
Nat Mulkey ${ }^{1}$ (D) \\ Received: 2 August 2020 / Accepted: 30 September 2020 / Published online: 8 October 2020 \\ (C) Academic Psychiatry 2020
}

Writing a patient's psychiatric note is truly a challenge, and I love it. Different from other specialties, the psychiatric note requires more subjective impressions from the author, with less pre-formed objective data (e.g., labs, test results) to rely on. High-quality patient documentation, including accurate, unbiased details of their medical and psychiatric history, goes far in helping to provide patients with cohesive care. It is not surprising that "documentation and communication" is a core clinical skill emphasized by undergraduate medical educators. The learning objectives for medical student psychiatric documentation include writing an accurate history, exam, daily progress updates, and overall diagnostic impression [1]. At the beginning of my medical training, with these objectives in mind, I thought all patient documentation would be a perfectly crafted retelling of subjective data, leading to an accurate diagnosis. Entering the clinical world as a medical student, I began to see how this ideal of clinical documentation stacked up against reality.

When I began to utilize patient charts, I came across a frustrating phenomenon. While reviewing background information for one of my patients, their chart stated they had bipolar disorder, anxiety, and depression. Though it was possible that this patient had a complex psychiatric history, this seemed inaccurate even with my basic understanding of diagnostic criteria. How could one individual have both bipolar disorder and unipolar depression? It turned out that there were many patient charts containing an array of psychiatric diagnoses that were not easily substantiated. The reason for this soon became clear to me, not from any formal didactic but by accident. While putting the finishing touches on one of my notes, I concluded that a patient likely had some form of "altered mental status," yet I did not feel I could give any more specifics without compromising accuracy. My preceptor informed me that I would need to change the diagnosis to "delirium"

Nat Mulkey

mulkeyna@bu.edu

1 Boston University Medical School, Boston, MA, USA because "altered mental status" was not a billable diagnosis. I thought to myself: what is a billable diagnosis?

When I got home, I started to investigate this question. Apparently, centralized diagnostic classification systems have been around since the late nineteenth century. The most recent iteration is the ICD-10 coding system. Using these codes, and using them well, is how physicians and healthcare systems get reimbursed. The ICD-10 provides many advantages including a uniform system of diagnosis and data that can be used for research [2]. However, this system has also been criticized for its collateral consequences, including the effects of directly tying these codes to physician pay. Critics argue that the ICD-10 and prior coding forms have led to incomplete and inaccurate information in documentation and little room for documenting a possible diagnosis verses a sure one [3]. I started to see a connection between this system and a new term I was hearing from my senior colleagues: "chart lore" [4]. As I continued to question the origin of certain diagnoses in patients' charts, I heard many responses along the lines of "oh that is just chart lore" or "that must be a chart diagnosis." These terms seem to refer to a loose diagnosis in documentation that may not be entirely accurate for the patient. Because of the stigma toward mental health conditions, the addition of a psychiatric chart diagnosis may carry more weight than that of a non-psychiatric diagnosis [5]. After seeing emphasis placed on accurate documentation in my medical school curriculum, this pattern of including uncertain potential diagnoses in patient documentation was hard to reconcile.

Diagnostic coding systems are not the only reason behind the gap I observed in learned documentation versus real documentation: the other crucial variable is time. As the learner, I am usually provided with ample time and space to complete my notes. I am also encouraged to explain my diagnostic thinking both for my own learning and for evaluation purposes. It is not surprising that I can produce a mindful and elaborative note when I am carrying one or two patients, as compared with the intern on my team who is carrying seven. Typically, the residents and interns I have worked with still produce thoughtful and accurate notes, but I have also seen many scenarios where time does not permit for this. 
At first, I struggled with how to approach the reality of patient documentation. Instead of the polished result of clinical thought, documentation can be a messy process due to time and system constraints. Initially I was disillusioned after learning about the monetary forces behind diagnosis and the lack of time and space for clinical postulating in notes. But after reviewing these realities of patient documentation, I have come to see our documentation system as exactly what it has always been: a tool. It is not gospel or the end-all be-all of a patient's care and summary. I would have liked for these realities of patient documentation to have been more explicitly emphasized in my undergraduate medical curriculum. Medical students do need to learn how to document in the ideal format, which includes describing and elaborating on our diagnostic thinking. Yet, we also need to learn the practical information about patient documentation and its understandable flaws. Medical schools should include curriculum about diagnostic coding systems and have transparent discussions with medical students about how much to rely on patient documentation. This information empowers us to be better users of this important clinical tool. Personally, after this exploration, I feel empowered to be a better user of patient documentation and a better clinician overall.

\section{Compliance with Ethical Standards}

Disclosures The author states that there is no conflict of interest.

\section{References}

1. Burke MJ, Campbell C, Dunstone D, et al. Clinical learning objectives guide for psychiatric education of medical students. Association of directors of medical student education in psychiatry. 2007. https:// www.admsep.org/pdf/templates/Clinical_LO_Guide_Outline_ Format_Nov_07.pdf. Accessed Jul 25th, 2020.

2. Topaz M, Shafran-Topaz L, Bowles KH. ICD-9 to ICD-10: evolution, revolution, and current debates in the United States. Perspect Health Inf Manag. 2013;10:1d.

3. Weiner MG. POINT: is international statistical classification of diseases and related health problems, 10th revision diagnosis coding important in the era of big data? Yes Chest. 2018;153(5):1093-5.

4. Yackel TR, Embi PJ. Copy-and-paste-and-paste. JAMA. 2006;296(19):2315-6. https://doi.org/10.1001/jama.296.19.2315-a.

5. Rössler W. The stigma of mental disorders: a millennia-long history of social exclusion and prejudices. EMBO Rep. 2016;17(9):1250-3. https://doi.org/10.15252/embr.201643041.

Publisher's Note Springer Nature remains neutral with regard to jurisdictional claims in published maps and institutional affiliations. 